\title{
Subpicosecond emission studies of bacterial reaction centers
}

\author{
P. Hamm ${ }^{\text {a }}$, K.A. Gray ${ }^{\text {b }}$, D. Oesterhelt ${ }^{b}$, R. Feick ${ }^{b}$, H. Scheer ${ }^{c}$ and W. Zinth ${ }^{\text {a }}$ \\ a Institut für Medizinische Optik der Ludwig-Maximilians-Universität München, München (Germany), ${ }^{b}$ Max-Planck-Institut für \\ Biochemie, Martinsried (Germany) and ${ }^{c}$ Botanisches Institut der Ludwig-Maximilians-Universität München, München (Germany)
}

(Received 18 August 1992)

Key words: Reaction center; Femtosecond spectroscopy; Fluorescence; Photosynthesis; (Rb. sphaeroides); (C. aurantiacus)

The spontaneous emission of reaction centers from native and mutated Rhodobacter sphaeroides and from wild type Chloroflexus aurantiacus is investigated by fluorescence up-conversion with high temporal resolution. The time constant of 0.9 ps previously observed in transient absorption experiments on wild type rcaction centers of Rhodobacter sphaeroides does not appear in the emission experiment. However, all investigated reaction centers display a biexponential decay of the emission with time constants in the $2 \mathrm{ps}$ to $25 \mathrm{ps}$ range. The experimental results are discussed within the frame of different reaction models including a possible sample heterogeneity or a transient electron transfer to the inactive pigment branch.

\section{Introduction}

The primary photosynthetic reaction stores light energy by an electrogenic charge transfer across a membrane bound pigment-protein complex called the reaction center (RC). The X-ray structure analysis of RC from two purple bacteria, Rhodopseudomonas (Rps.) viridis and Rhodobacter (Rb.) sphaeroides has shown that the pigments are arranged in two branches ( $A$ and B) related by an approximate $\mathrm{C}_{2}$-symmetry [1-6]. The branches start at the periplasmic side with two strongly interacting bacteriochlorophyll (BChl) molecules forming the special pair $P$ which acts as the primary electron donor. In succession, each branch contains a monomeric $\mathrm{BChl}$ molecule $\left(\mathrm{B}_{\mathrm{A}}, \mathrm{B}_{\mathrm{B}}\right)$, a bacteriopheophytin (BPhe) $\left(\mathrm{H}_{A}, \mathrm{H}_{B}\right)$ and ends with a quinone $\left(\mathrm{Q}_{A}\right.$, $\mathrm{Q}_{\mathrm{B}}$ ) at the cytoplasmic side. Spectroscopy has revealed that the two pigment branches are non-equivalent and that electron transfer proceeds predominantly via the A branch [7-9]. The two branches are also asymmetric in some structural aspects: (i) the lower disorder in the A branch indicates that it is more rigid [3]; (ii) $R b$. sphaeroides contains the polar amino-acid tyrosine M210 at a position near the special pair $P$ and the monomeric $\mathrm{BChl} \mathrm{B}_{\mathrm{A}}$, while the symmetry related position L181 on branch $B$ contains the nonpolar phenylalanine; (iii) the distance between the $\mathrm{BChl} \mathrm{B}$ and the

Correspondence to: W. Zinth, Institut für Medizinische Optik, Ludwig-Maximilians-Universität, Barbarastr. 16, 8000 München, Germany.
BPhe $\mathrm{H}$ is larger on the $\mathrm{B}$ branch [10]. Even greater differences exist between the two branches in the thermophilic green bacterium Chloroflexus aurantiacus. Leucine is found instead of tyrosine M210 and the monomeric $\mathrm{BChl} \mathrm{B}_{\mathrm{B}}$ on the $\mathrm{B}$-branch is most likely replaced by a $B P$ he [11].

The knowledge of the molecular structure and the ability to carry out site-directed mutagenesis and pigment exchanges make the reaction centers an ideal system to study structure-function relationships in an electron transfer system. The primary reactions are related to multiple absorption transients. At room temperature the native $\mathrm{RC}$ exhibits one time constant below 1 ps $(0.9$ ps in $R b$. sphaeroides and 0.65 in Rps. viridis) and other time constants of about 3 ps and 200 ps [12-17]. While it is generally accepted that the 200 ps process is related to the electron transfer from the $B$ Phe $H_{A}$ to the quinone $Q_{A}$, no general agreement has been obtained on the molecular interpretation of the other two time constants. At present, two basically different reaction models are discussed: [12-27] (i) In the stepwise electron transfer model the electron travels from the special pair $(P)$ within $\simeq 3$ ps to the monomeric $\mathrm{BChl} \mathrm{B}_{\mathrm{A}}$; in a second, faster step $(0.9 \mathrm{ps}$ or $0.65 \mathrm{ps}$ ) it reaches the bacteriopheophytin $\mathrm{H}_{\mathrm{A}}$. (ii) In the superexchange model the accessory bacteriochlorophyll $B_{A}$ is not a real electron carrier. The electron is transferred in one step from the special pair to the BPhe $\mathrm{H}_{\mathrm{A}}$. The 0.9 ps kinetic component must be related to some other process in this model like excited state vibrational relaxation of the special pair. Up to now the time resolved experiments on native $\mathrm{RC}$ gave 
no direct evidence for a possible involvement of the pigments on the $\mathrm{B}$ branch in the electron transfer process $[9,28]$.

In this article we describe time-resolved emission spectroscopy of RCs as an alternative method to transient absorption spectroscopy. A high time resolution was obtained by the technique of fluorescence up-conversion. By monitoring the fluorescence emission one observes selectively the emitting, i.e., the electronically excited states. There is no interference with absorbance changes of long-lived groundstate intermediates. As a consequence one can determine the decay of the excited electronic levels with higher precision. We will present data on native and mutated RCs from the purple bacterium $R b$. sphaeroides and on native RCs of the green bacterium Chloroflexus aurantiacus.

\section{Material and Methods}

Reaction centers from $R b$. sphaeroides strain R26.1 and from Chloroflexus aurantiacus Jfl-10 were prepared as described in Ref. 29 and Ref. 11, respectively. The construction of mutant strains and the purification of mutant RCs from $R b$. sphaeroides have been rcported in detail in Refs. 30, 31. Three different mutants were investigated here: (i) the tyrosine YM210 replaced by a phenylalanine (YM210 $\rightarrow$ F); (ii) the phenylalanine FL181 replaced by a tyrosine (FL181 $\rightarrow$ Y); (iii) the double mutant where both amino-acids are exchanged (YM210 $\rightarrow$ F, FL181 $\rightarrow$ Y). During the experiment the different RCs were kept at room temperature $(298 \mathrm{~K})$ in buffer. The concentration of the sample was adjusted to a transmission of $T \simeq 20 \%$ at $865 \mathrm{~nm}$, for a $1 \mathrm{~mm}$ light path. Total volumes of not less than $0.2 \mathrm{ml}$ per sample were used. The $\mathrm{RC}$ solutions were stirred in order to exchange the illuminated volume between two laser flashes and to avoid excitation of long-lived intermediates.

The experiments were performed with light pulses from a femtosecond near infrared laser-amplifier system operated at a repetition rate of $10 \mathrm{~Hz}$. Single pulses at $\lambda=850 \mathrm{~nm}$ with a duration of $\simeq 100 \mathrm{fs}$ were split off into an excitation (i) and an up-converting (ii) branch:

(i) In the excitation branch, the wavelength is converted to $865 \mathrm{~nm}$ by continuum generation and reamplification. The parameters of the exciting pulses were: wavelength $\lambda_{\text {exc }}=865 \mathrm{~nm}, \Delta \lambda=10 \mathrm{~nm}$, duration $t_{\mathrm{P}} \simeq$ $200 \mathrm{fs}$, energy $0.2 \mu \mathrm{J}$. The pulses were focused on the 1 $\mathrm{mm}$ sample cell by an $f=400 \mathrm{~mm}$ lens yielding a spot diameter of $700 \mu \mathrm{m}$. Under these conditions less than $10 \%$ of the $\mathrm{RC}$ in the irradiated volume absorbed a photon. At this low level of excitation double excitation of $\mathrm{RC}$ and reamplification of the emitted fluorescence by stimulated emission are negligible. Fluorescence light of the sample emitted in the forward direction was collimated by an $f=25 \mathrm{~mm}$ achromatic lens, spectrally filtered by an interference filter with $\lambda_{\max }=920$ $\mathrm{nm}$ and halfwidth (fwhm) of $\Delta \lambda=20 \mathrm{~nm}$. The wavelength of $920 \mathrm{~nm}$ was used in order to record only light emitted from the low energy transition of the special pair. The light was focused by an $f=40 \mathrm{~mm}$ achromatic lens through a polarizer onto a $1 \mathrm{~mm}$ thick $\mathrm{BBO}$ ( $\beta$-barium borate) crystal. The crystal was cut for colinear type II phase-matching where the fluorescence light is the extraordinary ray. Type II phase-matching was used to obtain a better suppression of second harmonic light generated by the up-converting pulse and to obtain uncritical phase-matching.

(ii) In the up-converting branch the short pulse at $850 \mathrm{~nm}$ was directly used at an energy of $3 \mu \mathrm{J}$ after the polarization was rotated by $90^{\circ}$. This pulse was suitably delayed and focused slightly into the BBO crystal with a spot diameter of $\simeq 1 \mathrm{~mm}$. Colincar beams were achieved by the polarizer since they have perpendicular polarization. During the illumination of the BBO crystal by the up-converting beam sum-frequency generation occurred between the fluorescence light (present at that time in the crystal) and up-converting photons. This up-converted light was spectrally filtered by interference filters $\left(\lambda_{\text {Max }} \simeq 441 \mathrm{~nm}, \Delta \lambda=8 \mathrm{~nm}\right)$, detected by a photomultiplier and recorded as a function of time delay between exciting and up-converting pulse. This signal reflected the temporal dependence of the emission from the sample. At the peak of the signal curve approx. 5 photoclectrons per single laser shot were detected. Therefore, extended data accumulation (2000-3000 single measurements per delay position) was necessary in order to obtain a sufficient dynamic range of the signal. Even without $\mathrm{RC}$ in the sample cuvette, a certain number of photons was detected. This delay-time-independent background (it was about $1 / 50$ of the peak signal) originated from incomplete blocking of the second harmonic of the up-converting light by the interference filters. In the displayed curves of the figures the background was subtracted. The experimental system was designed for a time resolution in the subpicosecond range. Under the experimental conditions used a width of the instrumental response function of $400-500 \mathrm{fs}$ was measured. This width originated from the group velocity dispersion in the $1 \mathrm{~mm}$ sample cell and the BBO crystal and from the duration of the exciting and up-converting light pulses. The time resolution was sufficient to measure emission decay times well below 1 ps.

It is general practice to perform up-conversion experiments with subpicosecond time resolution at high repetition rates. This allows low levels of excitation in the sample, preventing reamplification of the emitted light but still yields a sufficient number of up-converted photons. However, the long duration of reaction cycles in photosynthetic RC (up to several milliseconds) limits 


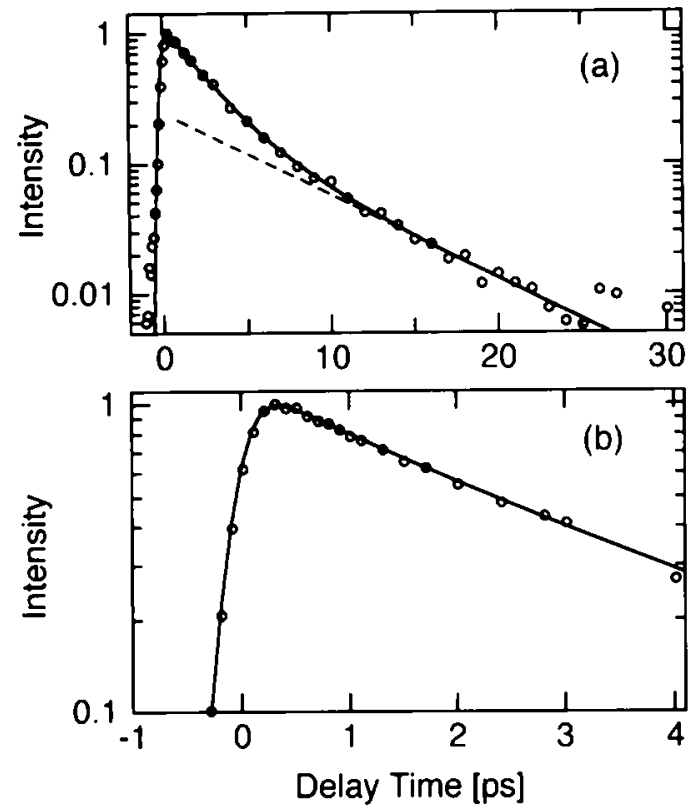

Fig. 1. The $\mathrm{P}^{*}$ spontaneous emission of unmodified RCs of R26.1. The data points are represented by circles, the solid line is a model function where a sum of exponentials is convoluted with a Gaussian function. (a) Decay of the emission for delay times up to 30 ps. A biexponential behaviour is evident. Time constants of $2.3 \mathrm{ps}$ and $7 \mathrm{ps}$ are used to model the decay. (b) The same signal curves as in (a) in an expanded view. There is no need of a kinetic component with a faster time constant (i.e., the 0.9 ps kinetic component which was observed in transient absorbance measurements).

the repetition rates. The $10 \mathrm{~Hz}$ repetition rate applied in our experiments and used in combination with a sample stirring minimized the accumulation of photoproducts, even for the small sample volumes available.

\section{Results}

In a first experiment, the emission of reaction centers from $R b$. sphaeroides $\mathrm{R} 26.1$ was investigated. Fig. 1 shows the up-conversion signal on a logarithmic scale as a function of the delay time. The experimental data are displayed as filled circles. The solid curve is a model function where a sum of exponentials is convo- luted with a Gaussian function of suitable halfwidth (the Gaussian is used to simulate the instrumental response function). The data can not be explained by a monoexponential decay: At first the emission of the sample rises with the exciting pulse. Afterwards it decays fast over the first order of magnitude but slower at later delay times (see Fig. 1a). From the modelling of the data the slow decay time is determined as 7 ps \pm 2 ps while the first initial decay is related to a 2.3 ps \pm 0.4 ps time constant. The biexponential model function with a ratio of the amplitudes of $A(7 \mathrm{ps}) / A$ $(2.3 \mathrm{ps})=0.25 \pm 0.1$ fits the data points very well. However, one can not rule out other kinetic components with longer life times and with very small amplitudes. Of special interest is the signal during the first picosecond (see Fig. 1b). In this time range, also, the fit with the two time constants of 2.3 ps and 7 ps used above is satisfactory. If a $0.9 \mathrm{ps}$ time constant is included in the fit, its amplitude is less than $8 \%$ with a high amplitude uncertainty and no significant improvement of the fit.

How does the biexponential fluorescence emission compare with published data? In a previous publication we had shown that the transient stimulated emission (gain) due to the population of the excited electronic state $\mathrm{P}^{*}$ of $\mathrm{RC}$ from $R b$. sphaeroides could be fitted within the signal to noise ratio by a single exponential with a time constant of 3.5 ps [13]. In that experiment we could not rule out the existence of a component with a longer lifetime. In view of our present data, the time constant of $3.5 \mathrm{ps}$ appears to be an averaged value which results from a fit of the biexponential decay by a monoexponential model function. A reevaluation of the stimulated emission data of $R b$. sphaeroides using the time constants determined in the emission experiment yielded amplitudes of the 7 ps kinetic component of up to $25 \%$ with a large scattering of the amplitude values from experiment to experiment. Recently, a biexponential gain (stimulated emission) was reported in the literature by Vos et al. [27] while slower kinetic components in the fluorescence emission were observed by Holzwarth et al. [32].

Non-monoexponential decays of the emission are also evident in the mutants YM210 $\rightarrow \mathrm{F}, \mathrm{FL} 181 \rightarrow \mathrm{Y}$

TABLE I

Measured decay time constants $\tau_{1}$ and $\tau_{2}$ of the spontaneous emission and microscopic rate constants $\sigma_{i}$ determined on the basis of Scheme Ib (see text for details and symbols)

\begin{tabular}{|c|c|c|c|c|c|c|}
\hline & $\begin{array}{l}\text { Fast component } \\
\tau_{1} \text { (ps) }\end{array}$ & $\begin{array}{l}\text { Slow component } \\
\tau_{2}(\mathrm{ps})\end{array}$ & $\eta=\left(\mathrm{A}\left(\tau_{2}\right) / \mathrm{A}\left(\tau_{1}\right)\right)$ & $\begin{array}{l}\sigma_{x}^{-1} \\
(\mathrm{ps})\end{array}$ & $\begin{array}{l}\sigma_{N}{ }^{1} \\
(\mathrm{ps})\end{array}$ & $\begin{array}{l}\sigma_{\mathrm{N}^{\prime}}^{\prime} \\
(\mathrm{ps})\end{array}$ \\
\hline Rb. sphaeroides R26.1 & 2.3 & 7 & 0.25 & 3.2 & 13 & 4.8 \\
\hline Rb. sphaeroides $\mathrm{YM} 210 \rightarrow \mathrm{F}$ & 6.1 & 26 & 1.37 & 18 & 29 & 9.0 \\
\hline Rb. sphaeroides FL181 $\rightarrow \mathrm{Y}$ & 2.1 & 15 & 0.06 & 2.8 & 10.3 & 11.2 \\
\hline \multicolumn{7}{|l|}{$R b$. sphaeroides $\mathrm{YM} 210 \rightarrow \mathrm{F}$} \\
\hline $\mathrm{FL181} \rightarrow \mathrm{Y}$ & 3.5 & 18 & 0.59 & 8.8 & 11 & 7.1 \\
\hline Chloroflexus aurantiacus & 2.7 & 9.1 & 1.0 & 5.9 & 14 & 4.2 \\
\hline
\end{tabular}




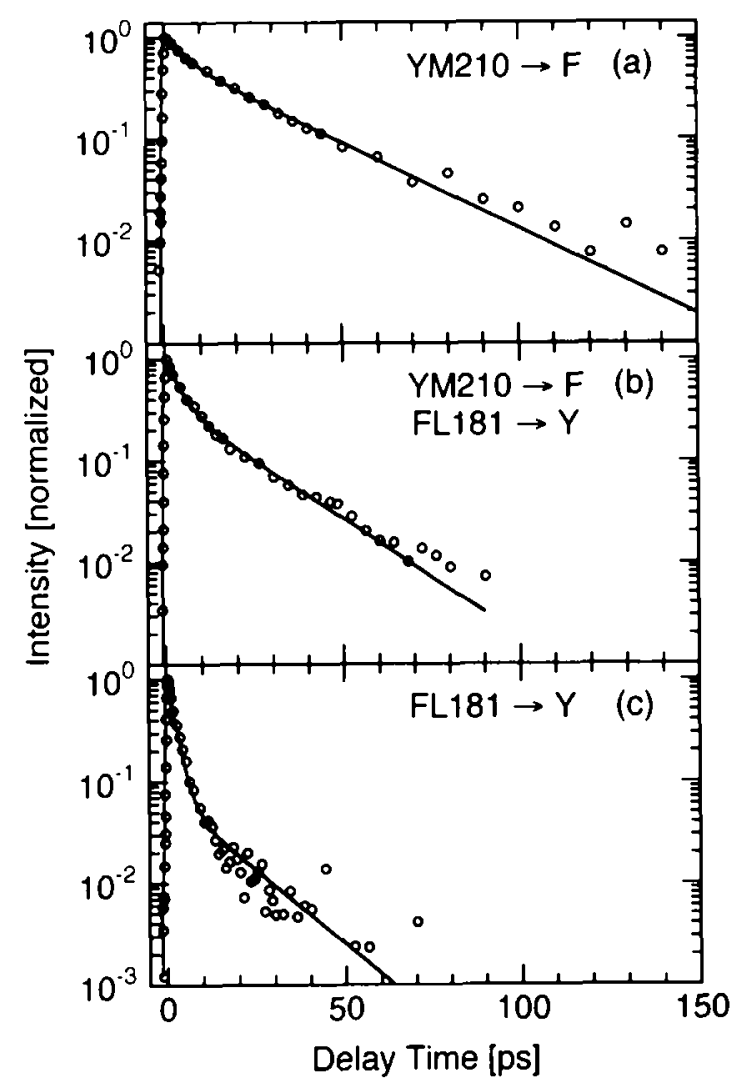

Fig. 2. Spontaneous emission data from the single site mutant RCs $\mathrm{YM} 210 \rightarrow \mathrm{F},(\mathrm{a})$ and $\mathrm{FL} 181 \rightarrow \mathrm{Y},(\mathrm{c})$, and the double mutant YM210 $\rightarrow \mathrm{F}+\mathrm{FL} 181 \rightarrow \mathrm{Y},(\mathrm{b})$. In each case a biexponential decay was required to model the data points. The values of the time constants and amplitude ratios are summarized in Table I, left part.

and YM210 $\rightarrow$ F, FL181 $\rightarrow$ Y (Fig. 2). A biexponential fit yielded time constants between 2.1 ps and 6.1 ps for the fast process and between 15 ps and 26 ps for the slow process at different amplitude ratios (see Table I, left part). Again, a qualitative agreement is found with published transient absorption data [33,34].

Reaction centers of Chloroflexus aurantiacus show a biexponential decay with time constants of $\tau=2.7 \mathrm{ps}$

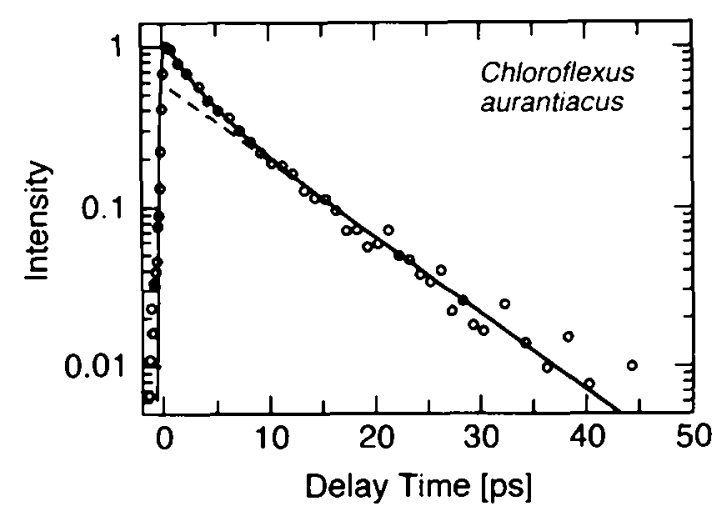

Fig. 3. Spontaneous emission of RC of Chloroflexus aurantiacus. Two kinetic components ( $2.7 \mathrm{ps}, 9.1 \mathrm{ps}$ ) with nearly the same amplitude were used to fit the data. and $\tau=9.1 \mathrm{ps}$ and an amplitude ratio of $\mathrm{A}_{2} / \mathrm{A}_{1}=1$ (Fig. 3). One does not find a detectable contribution from a longer lived kinetic component. These observations complement published absorption experiments at various low temperatures where a biexponentiality of the stimulated emission was observed [35]. Recent room temperature data recorded in the gain region could be fitted by a single exponential decay with $\tau=7.1 \pm 0.5$ ps [36]. However, the better signal-to-noise ratio of the present investigation leads to the conclusion that the 7.1 ps time constant originates from a monoexponential fit of a biexponential decay. Time-resolved emission experiments on Chloroflexus aurantiacus were performed previously by Müller et al. [37]. In these experiments, carried out with high repetition rates in the $\mathrm{MHz}$ regime and a width of the instrumental response function of $30 \mathrm{ps}$, a series of time constants was found. Under the conditions called 'open reaction centers' and excitation at $850 \mathrm{~nm}$ time constants of 7 ps, $20 \mathrm{ps}, 110 \mathrm{ps}$ and $1.1 \mathrm{~ns}$ were reported. In view of their limited time resolution, these experiments do not conflict with the time constants found in our experiments. However, the observation of the 20 ps component by Müller et al., not detected otherwise, may be due to the specific experimental conditions.

The salient results of our time-resolved fluorescence up-conversion experiments can be summarized as follows: (i) There is no indication of a fast, namcly a 0.9 ps kinetic component. (ii) The emission decays biexponentially with time constants $\tau_{\mathrm{i}}>2$ ps as shown in Table I. (iii) The specific values of the time constants and the amplitude ratios depend strongly on the type of reaction center.

\section{Discussion}

The absence (within our signal to noise ratio) of the 0.9 ps kinetic component in the emission data of reaction center from $R b$. sphaeroides supplements the data obtained from transient absorption spectroscopy performed in the gain region [13,14]. Both types of experiments agree well in this respect. The arguments presented in Ref. 14 on the interpretation of the gain data can be extended to the emission experiment: if there is a second excited electronic level of $\mathrm{P}^{*}$ formed within 0.9 ps it must have specific spontaneous emission properties which are not consistent with the idea of an excited state vibrational relaxation related to the $0.9 \mathrm{ps}$ kinetic component. Because of this finding, we will concentrate on reaction models where the 0.9 ps kinetic component is related to a later process (i.e., the reaction step from the $B C h l B_{A}$ to the $B P h e H_{A}$ ).

On the other hand, the spontaneous emission curves cannot be fit monoexponentially on the time scale of 2-20 ps, but need a second, slower component. We shall now discuss possible mechanisms leading to this 


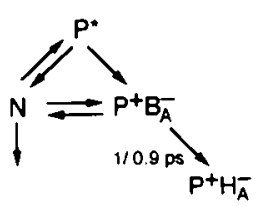

(a)

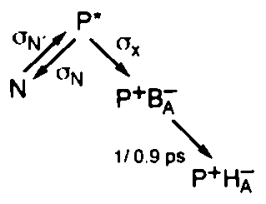

(b)

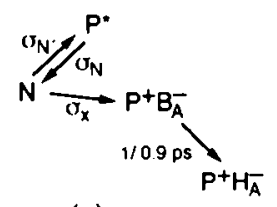

(c)
Scheme I. Possible models of the primary processes in photosynthetic reaction centers for a homogeneous $\mathrm{RC}$ preparation.

biexponentiality. It is well established that the room temperature electron transfer reactions can be described by non-adiabatic electron transfer theory [22]; in this case, the population of the intermediate states and, as a consequence, the transient emission and the absorption data can be described by a rate equation system. The analysis of rate equation systems yields as a basic result that the number of intermediate states is not smaller than the number of exponentials observed [13]. Until now transient absorption spectroscopy has proven the existence of four intermediate states by the observation of the time constants $\tau_{1}=0.9 \mathrm{ps}, \tau_{2}=3 \mathrm{ps}$, $\tau_{3}=200 \mathrm{ps}$ and $\tau_{4}={ }^{\prime} \infty$ '. Duc to the independent emission experiment the 3 ps process in unmodified reaction centers has now to be split into two processes with time constants given above (Table I). This supports the idea of an additional intermediate state or some kind of heterogeneity.

In the latter case one would deal with two subcomponents of the sample having different primary electron transfer reactions. There are some indications of heterogeneities for different reaction centers: For Chloroflexus aurantiacus a heterogeneity was revealed by isoelectric focusing showing three RC fractions (Ref. 38 and Feick, R., personal communication). For $R b$. sphaeroides it is known that prolonged illumination reveals an RC fraction which has a slow (minutes) recovery time of the light induced bleaching (Scheer, H., Beese, D. and Struck, A., unpublished data). However, it is not clear how these heterogeneities arise or if they are related to the primary reactions.

For a homogeneous sample one has to assume that the longer emission decay time is related to a new state not yet contained in the reaction models mentioned in the introduction. In this case, the additional time constants can not be explained by introducing only new connections (e.g., back-transfer rates or parallel reactions) in the known set of the intermediate states. The experimental observation of this new state (we call it $\mathrm{N}$ ) in the emission experiment indicates that $\mathrm{N}$ must be a state coupled directly to $\mathbf{P}^{*}$. In Scheme Ia $\mathbf{N}$ is introduced into the former model allowing all possible reaction paths. If $\mathrm{N}$ is not an electronically excited state, i.e., $\mathrm{N}$ is not emitting, the back-reaction from $\mathrm{N}$ to $P^{*}$ is necessary to explain the biexponential decay. The back-reaction from $\mathrm{P}^{+} \mathrm{B}_{\mathrm{A}^{-}}$to $\mathrm{P}^{*}$ can be neglected; otherwise the 0.9 ps kinetic component should be observed clearly in the emission experiments. In more detail: If one assumes that the energy of $\mathrm{P}^{+} \mathrm{B}_{\mathrm{A}^{-}}$ is approx. $500 \mathrm{~cm}^{-1}$ below that of $P^{*}$ one can estimate the back-reaction from $\mathrm{P}^{+} \mathrm{B}_{\mathrm{A}^{-}}$to $\mathrm{P}^{*}$ to proceed 10 times more slowly than the forward reaction, i.e., with a time constant of about $30 \mathrm{ps}$ [22]. The combination of this time constant with the very fast secondary transfer from $\mathrm{P}^{+} \mathrm{B}_{\mathrm{A}}$ to $\mathrm{P}^{+} \mathrm{H}_{\mathrm{A}^{-}}$leads to a small amplitude of the 0.9 ps kinetic component in the emission decay of $\mathrm{P}^{*}$ of less than $5 \%$. This value is within the experimental uncertainty given above.

Since it is not possible to evaluate the other unknown reaction rates on the basis of the experimental data (without additional assumptions), we want to concentrate on two limiting cases (Scheme Ib and Ic) of the general model in Scheme la by neglecting some of the reaction paths. In Scheme $\mathrm{Ib}, \mathrm{N}$ is a 'parking state' coupled only to $\mathrm{P}^{*}$ with rate constants $\sigma_{\mathrm{N}}$ and $\sigma_{\mathrm{N}}^{\prime}$. A reaction rate $\sigma_{\mathrm{x}}$ is used from $\mathrm{P}^{*}$ into the first radical pair (presumably $\mathrm{P}^{+} \mathrm{B}_{\mathrm{A}}$ ). Scheme $\mathrm{Ic}$ is a purely sequential reaction model. In both cases the microscopic rates $\sigma_{\mathrm{i}}$ can be deduced from the observed decay rates $\gamma_{\mathrm{i}}=1 / \tau_{\mathrm{i}}$ and the amplitude ratios $\eta=A$ (slow) $/ A$ (fast) if it is assumed that the new state $\mathrm{N}$ is not emitting. A numerical evaluation of the model of Scheme Ic (sequential model) shows that the forward reaction $\sigma_{x}$ is slowed down by a factor of 2 and unreasonable spectral propertics of state $\mathrm{P}^{+} \mathrm{B}_{\mathrm{A}^{-}}$result. Because of this fact we favour the model shown in Scheme Ib where $\mathbf{N}$ is a non-emitting 'parking state'. The values

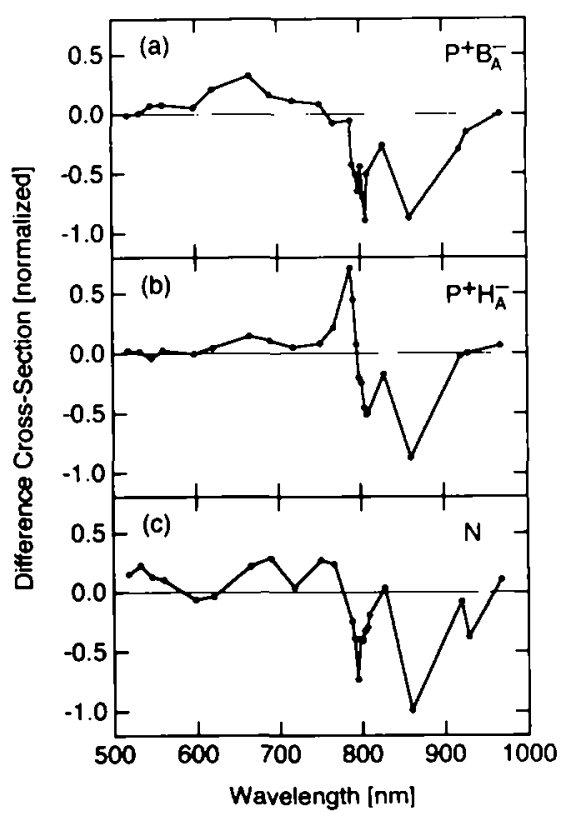

Fig. 4. Difference spectra of the intermediate states $P^{+} B_{A^{-}}, P^{+} H_{A^{-}}$ and $\mathrm{N}$ (see Scheme Ib) for RC of unmodified $R b$. sphaeroides. The spectra are calculated from transient absorbance data [12,13] which were fit with four time constants $(0.9 \mathrm{ps}, 2.3 \mathrm{ps}, 7 \mathrm{ps}, 200 \mathrm{ps})$ under the assumption of the reaction model in Scheme Ib. The experimental error in the spectra is approx. 0.2. 
of the microscopic rates of all samples calculated according to Scheme Ib are summarized in Table I.

Of special interest are the spectral properties of the intermediate $\mathbf{N}$. A reevaluation of previous transient absorbance data of $R b$. sphaeroides RC (Refs. 12, 13 and Lauterwasser, C., Finkele, U., personal communication) using the new set of time constants and Scheme $\mathrm{Ib}$ allows us to estimate the difference spectra of all intermediates involved (i.e., the difference between the absorption cross-section of the corresponding intermediate and that of the ground state RCs). The resulting spectra of $\mathrm{P}^{-} \mathrm{B}_{\mathrm{A}}, \mathrm{P}^{+} \mathrm{H}_{\mathrm{A}}$ and $\mathrm{N}$ are displayed in Fig. 4. The spectrum of the state $\mathrm{N}$ shows a bleaching around $860 \mathrm{~nm}\left(\mathrm{Q}_{\mathrm{y}}(\mathrm{P})\right)$, at $800 \mathrm{~nm}\left(\mathrm{Q}_{\mathrm{y}}(\mathrm{B})\right)$ and at 600 $\mathrm{nm}\left(\mathrm{Q}_{\mathrm{x}}(\mathrm{P}, \mathrm{B})\right)$. A broad transient absorbance increase occurs around $650 \mathrm{~nm}$ in the range of the absorbance bands of $\mathrm{BChl}$ or BPhe anions. The bleaching in the region of $Q_{y}(B)$ is blue-shifted by approx. $5 \mathrm{~nm}$ as compared to the corresponding band of the state $\mathrm{P}^{-} \mathrm{B}_{\mathrm{A}^{-}}$. These findings support the idea that $\mathrm{N}$ might be a transiently populated radical pair state $\mathrm{P}^{+} \mathrm{B}_{\mathrm{B}}$ on the 'inactive', i.e., $B$ branch. The energy of such a radical pair state and as a consequence the reaction rates to it should be sensitive to the modification of the polarity in the surroundings of the special pair and the BChl monomers.

The evaluation of the emission data on mutated RC according to Scheme Ib indicates that the replacement of tyrosine $\mathrm{M} 210$ by phenylalanine on the A-branch slows down strongly the rate $\sigma_{\mathrm{x}}$ on the A-branch and to a lesser extent the rate $\sigma_{N}$ to the parking level $N$. Replacement of phenylalanine L181 by a tyrosine speeds up both forward rates but slows down the rate $\sigma_{N}$, of the back reaction. These results agree with the idea that the polarity in the surroundings of the chromophores modulates the electron transfer. Increasing the polarity speeds up the electron transfer reaction predominantly in the branch where the polarity was increased. This is also consistent with the idea of $\mathrm{N}$ being a radical pair on the $B$ branch, presumably $\mathrm{P}^{+} \mathrm{B}_{\mathrm{B}}$. In the reaction centers of Chloroflexus aurantiacus the model of Scheme lb yields rates to and from the parking level $\mathrm{N}$ which are similar to those of reaction centers of $R b$. sphaeroides $\mathrm{R} 26.1$. The reaction rate to $\mathrm{P}^{+} \mathrm{B}_{\mathrm{A}^{-}}$seems to be slower by a factor of 2 . This difference could be due to the fact that in Chloroflexus aurantiacus a leucine is at the position related to YM210 of $R b$. sphaeroides.

In a very simplified interpretation one could also relate the observed kinetics to the modification of the energy of the radical pair states $\mathrm{P}^{+} \mathrm{B}_{\mathrm{A}^{-}}$or $\mathrm{P}^{+} \mathrm{B}_{\mathrm{B}^{-}}$by the changed amino-acid composition. From the ratio of rates $\sigma_{\mathrm{N}}$ and $\sigma_{\mathrm{N}}^{\prime}$ one could estimate the energy differences between $N$ and $\mathrm{P}^{*}$. Under the assumption of nondegenerate states $\mathrm{N}$ and $\mathrm{P}^{*}$ the values in Table I (right side) indicate that $\mathrm{N}$ lies above $\mathrm{P}^{*}$ for all sam- ples except the Rb. sphaeroides mutant protein FL181 $\rightarrow Y$. As a consequence, the biexponentiality should decrease at low temperatures. According to the experimental data available to date $[27,35]$ this temperature dependence is not observed experimentally. It should be noted, however, that the low temperature experiments were performed in the presence of cryoprotectors such as glycerol or polyvinylalcohol (PVA) which are known to modify the primary reaction kinetics by themselves [39]. In addition, it is not known whether the energetics of the $\mathrm{RC}$ are really temperature independent. Nevertheless some of the very crude assumptions made in Scheme Ib, as, for example, the non-degeneracy of the states or the omission of some reaction paths may not be justified and need further studies.

\section{Conclusions}

The fluorescence emission of reaction centers of $R b$. sphaeroides $\mathrm{R} 26.1$ and $R b$. sphaeroides mutants $(\mathrm{YM} 210 \rightarrow \mathrm{F}, \quad \mathrm{L} 181 \rightarrow \mathrm{Y}, \quad \mathrm{YM} 210 \rightarrow \mathrm{F}+\mathrm{FL} 181 \rightarrow \mathrm{Y})$ and wild type reaction centers of Chloroflexus aurantiacus was studied with high time resolution. The emission data give no indication of the 0.9 ps time constant found previously in transient absorption measurements [12-14]. However, the data clearly show a biexponential decay of the emission at later times. One possible explanation would be a functional heterogeneity of the reaction center preparation which could influence the primary electron transfer. Alternatively, the biexponentiality can be described in the frame of a homogeneous reaction center preparation where a new intermediate state is introduced. One possible assignment for the new state would be a radical pair state involving a transient electron transfer towards the B branch. Under this assumption the high asymmetry of the charge transfer process would be due to both an asymmetry of the initial charge separation and a very slow electron transfer from the monomeric bacteriochlorophyll $B_{B}$ to its ncighbouring bactcriopheophytin $\mathrm{H}_{\mathrm{B}}$.

\section{Acknowledgements}

The authors gratefully acknowledge valuable discussions and continuous support by W. Kaiser and experimental help by $\mathrm{C}$. Lauterwasser. This work was supported by the Deutsche Forschungsgemeinschaft, SFB 143.

\section{References}

1 Deisenhofer, J., Epp, O., Miki, K., Huber, R. and Michel, H. (1984) J. Mol. Biol. 180, 385-398.

2 Deisenhofer, J., Epp, O., Miki, K., Huber, R. and Michel, H. (1985) Nature 318, 618-622.

3 Deisenhofer, J. and Michel, H. (1989) EMBO J. 8, 2149-2170. 
4 Allen, J.P., Feher, G., Yeates, T.O., Komiya, H. and Rees, D.C. (1987) Proc. Natl. Acad. Sci. USA 84, 5730-5734.

5 Allen, J.P., Feher, G., Yeates, T.O., Komiya, H. and Rees, D.C. (1987) Proc. Natl. Acad. Sci. USA 84, 6162-6166.

6 Chang, C.H., El-Kabbani, O., Tiede, D., Norris, J. and Schiffer, M. (1991) Biochemistry 30, 5352-5360.

7 Zinth, W., Knapp, E.W., Fischer, S.F., Kaiser, W., Deisenhofer, J. and Michel, H. (1985) Chem. Phys. Lett. 119, 1-4.

8 Kirmaier, C., Holten, D. and Parson, W.W. (1985) Biochim. Biophys. Acta 810, 33-48.

9 Michel-Beyerle, M.E., Plato, M., Deisenhofer, J., Michel, H., Bixon, M. and Jortner, J. (1988) Biochim. Biophys. Acta 932, 52-70.

10 El-Kabbani, O., Chang, C.H., Tiede, D., Norris, J., and Schiffer, M. (1991) Biochemistry 30, 5361-5369.

11 Shiozawa, J.A., Lottspeich, F., Oesterhelt. D. and Feick, R. (1989) Eur.J.Biochem. 180, 75-84.

12 Holzapfel, W., Finkele, U., Kaiser, W., Oesterhelt, D., Scheer, H., Stilz, H.U. and Zinth. W. (1989) Chem. Phys. Lett. 160, 1-7.

13 Holzapfel, W., Finkele, U., Kaiser, W., Oesterhelt, D., Scheer, H., Stilz, H.U. and Zinth, W. (1990) Proc. Natl. Acad. Sci. USA 87, 5168-5172.

14 Dressler, K., Umlauf, E., Schmidt, S., Hamm, P., Zinth, W., Buchanan, S. and Michel, H. (1991) Chem. Phys. Lett. 183, 270-276.

15 Woodbury, N.W., Becker, M., Middendorf, D. and Parson, W.W. (1985) Biochemistry 24,7516-7521.

16 Martin, J.L., Breton, J., Hoff, A.J., Migus, A. and Antonetti, A. (1986) Proc. Natl. Acad. Sci. USA 83, 957-961.

17 Breton, J., Martin, J.L., Migus, A., Antonetti, A. and Orszag, A. (1986) Proc. Natl. Acad. Sci. USA 83, 5121-5125.

18 Haberkorn, R., Michel-Beyerle, M.E. and Marcus, R.A., (1979) Proc. Natl. Acad. Sci. USA 76, 4185-4188.

19 Marcus, R.A. (1987) Chem. Phys. Lett. 133, 471-477.

20 Parson, W.W., Warshel, A., Creighton, C. and Norris, J.R. in (1988) The Photosynthetic Bacterial Reaction Center. Structure and Dynamics (Breton, J. and Vermeglio, A., eds.), pp. 309-317, Plenum Press, New York.

21 Bixon, M., Michel-Beyerle, M.E. and Jortner, J. (1988) Isr. J.Chem. 28, 155-168.

22 Bixon, M., Jortner, J. and Michel-Beyerle, M.E. (1991) Biochim. Biophys. Acta 1056, 301-317.
23 Kuhn, H. (1986) Phys. Rev. A 34, 3409-3425.

24 v.Kitzing, E. and Kuhn, H. (1990) J. Phys. Chem. 94, 1699-1702.

25 Fleming, G.R., Martin, J.L. and Breton, J. (1988) Nature 333, 190-192.

26 Chang, C.-K., DiMagno, T.J., Chen, L.X.-Q., Norris, J.R. and Fleming, G.R. (1991) Proc. Natl. Acad. Sci. USA 88, 11202-11206.

27 Vos, M.H., Lambry, J.-C., Robles, S.J., Youvan, D.C., Breton, J. and Martin, J.L. (1991) Proc. Natl. Acad. Sci. USA 88, 8885-8889.

28 Tiede, D.M., Kellogg, E.C., Kolaczkowski, S. and Wasielewski, M.R. (1990) in Current Research in Photosynthesis (Baltscheffsky, M., ed.), Vol. I, pp. 129-132, Martinus Nijhoff, Dordrecht.

29 Beese, D., Steiner, R., Scheer, H., Angerhofer, A., Robert, B. and Lutz, M. (1987) Photochem. Photobiol. 47, 293-304.

30 Gray, K.A., Farchaus, J.W., Wachtveitl, J., Breton, J. and Oesterhelt, D. (1990) EMBO J. 9 2061-2070

31 Gray, K.A., Wachtveitl, J. and Oesterhelt, D (1992) Eur. J. Biochem., in press.

32 Müller, M.G., Griebenow, K. and Holzwarth, A.R., (1991) Biochim. Biophys. Acta 1098, 1-12.

33 Finkele, U., Lauterwasser, C., Zinth, W., Gray, K.A. and Oesterhelt, D. (1990) Biochemistry 29, 8517-8521.

34 Chang, C.-K., Chen, L.X.-Q., DiMagno, T.J., Hanson, D.K., Nance, S.L., Schiffer, M., Norris, J.R. and Fleming, G.R. (1991) Chem. Phys. Lett. 176, 366-372.

35 Martin, J.L., Lambry, J.C., Ashokkumar, M., Michel-Beyerle, M.E., Feick, R. and Breton, J. (1990) in Springer Series in Chemical Physics, Ultrafast Phenomena VII (Harris, C.B., Ippen, E.P., Mourou, G.A. and Zewail, A.H., eds.), Vol. 53, pp. 524-528, Springer, Berlin.

36 Becker, M., Nagarajan, V., Middendorf, D., Parson, W.W., Martin, J.E. and Blankenship, R.E. (1991) Biochim. Biophys. Acta $1057,299-312$.

37 Schweitzer, G., Hucke, M., Griebenow, K., Müller, M.G. and Holzwarth, A.R. (1992) Chem. Phys. Lett. 190, 149-154.

38 Feick, R., Martin, J.L., Breton, J., Volk, N., Scheidel, G. Langenbacher, T., Urbano, C., Ogrodnik, A. and Michel-Beyerle, M.E. (1992) in Springer Series in Biophysics (Michel-Beyerle, M.E., ed.), Vol. 6, pp. 181-188 Springer, Berlin.

39 Aumeier, W., Thesis, Technische Universität München (1990). 